\title{
A Dynamic Topology based PEGASIS and DSN Routing Protocol in Mobility Wireless Sensor Network
}

\author{
Kiran Rani \\ Department of Electronics \& Communication \\ Engineering \\ SBSSTC Ferozepur 152004 \\ Punjab, India
}

\author{
Amit Grover \\ Department of Electronics \& Communication \\ Engineering \\ SBSSTC Ferozepur 152004 \\ Punjab, India
}

\begin{abstract}
PEGASIS and DSN Routing protocol (PDORP) is one of the heuristic enhancement techniques and a subset subject of swarm knowledge. PDORP depends on recreating the idea of how water drops consolidate to shape waterways and streams thusly join to join the Sea by choosing the most limited way in light of heights of the land through which they stream. An alteration in the PDORP calculation is made to accomplish the goals of the exploration in which a vitality limit set for every hub. In the event of the proposed approach with the low vitality in the subset of system the neighbour hubs of the subset can cover the zone of the kicked the bucket hub. The proposed approach is characterized on the premise of Ant Colony Optimization utilizing which the calculation turn out to be more vitality proficient. Thusly the information dropped because of the substitution of the subset might be diminished so there is a decrease in the vitality dispersal. Utilizing this approach the information dropped and other Quality parameters are likewise enhanced like, deferral, load and throughput and so forth as characterized in results and talk.
\end{abstract}

\section{Keywords}

WSN, MWSN, PDORP

\section{INTRODUCTION}

Wireless sensor arrange (WSN) alludes to a gathering of spatially scattered and committed sensors for checking and recording the physical states of the earth and sorting out the gathered information at a focal location[1]. WSNs measure the natural conditions like temperature, contamination levels, sound, mugginess, weight, wind speed and bearing, and so on. The WSN is worked of "hubs" - from a couple to a few hundreds or even thousands, where every hub is associated with one (some of the time a few) sensors[2]. Each such sensor organize hub has a few sections: a radio handset with an outside receiving wire or association with an interior reception apparatus, an electronic circuit for interfacing with the sensors and a vitality source, a microcontroller, as a rule a battery or an inserted type of vitality harvesting[3]. A sensor hub may differ in size from that of a shoebox down to the extent of a grain of clean, albeit working "bits" of honest to goodness infinitesimal measurements were made. The cost of sensor hub is also factor. It might extend from a couple to several dollars, contingent upon the many-sided quality of the individual sensor hub. Size and cost requirements on sensor hubs bring about relating imperatives on assets, for example, memory, vitality, interchanges data transfer capacity and computational speed [4]. The topology of the Wireless Sensor Networks can fluctuate from a straightforward star system to a progressed multi-jump remote work network [5].
PDORP is one of the heuristic improvement strategy and a subset theme of swarm knowledge. PDORP depends on recreating the idea of how water drops consolidate to frame waterways and streams thus consolidate to join the Sea by choosing the most limited way in view of elevations of the land through which they flow [6]. During the time spent stream arrangement, the water drops are continually spilling out of higher elevation position to lower height positions. Since, the slant of the two positions is all the more, then the water spilling out of higher positions to lower positions disintegrate and convey the dissolved soil to be kept in the lower positions[7]. By this store the height of the lower position get expanded. Likewise most brief way is framed from higher to lower position. The procedure of PDORP basically comprise of two phases viz., Initialization stage and River arrangement organize. In instatement arrange, three distinct positions (called water drop creating positions or Source (S), moderate positions (I), and goal (D) or ocean) are introduced. Every one of these positions are spoken to with various height esteem ( $\mathrm{S}$ and I are spoken to with positive elevation qualities and D is spoken to with Zero). The water drop producing positions dependably creates water drops. The middle of the road positions gets the water drops from source and forward towards the Sea. In waterway arrangement organize, the stream is made between drop creating positions and Sea utilizing the iterative procedure having the capacities select-Forward-Position (), move-Drops (), erode-Path (), and add-Sediments (). The iterative procedure is rehashed until either all drops take after a similar way or fulfilling the other completion conditions, for example, predetermined number of emphases, restricted execution time[8][9]. There is likeness an amongst PDORP and information gathering forms in WSN. In PDORP, the source (drop producing) positions create water drops and these water drops are intrigued to meet the goal or Sea. Essentially, in WSN information accumulations prepare, the sensor hubs create the information and this information is intrigued to achieve the base station [10]. Consequently, the sensor information act like water drops, the source positions like sensor hubs, and base station as Sea. The drops are joined and spills out of source to ocean to shape the streams in light of elevation estimation of position in PDORP [11]. Similarly to forward the information in WSNs, the sensor hubs can frame a way to the construct station situated in light of jump number and lingering vitality.

\section{RELATED STUDY}

Koppala Guravaiah et al (2015) projected a replacement mechanism for knowledge assortment and routing supported watercourse Formation Dynamics. The projected algorithmic program is termed as PDORPMRP: watercourse Formation Dynamics primarily based Multi-hop Routing Protocol. In Wireless sensing element networks, sensing element nodes 
sense the info from setting in keeping with its practicality and forwards to its base station. This method is termed knowledge assortment and it's done either direct or multi-hop routing. In direct routing, each sensing element node directly transfers its perceived knowledge to base station that influences the energy consumption from sensing element node thanks to the so much distance between the sensing element node and base station. In multi-hop routing, the perceived knowledge is relayed through multiple nodes to the bottom station, it uses less energy.

Gyanendra Prasad Joshi et al. (2013) delineated the benefits of psychological feature radio wireless sensing element networks, the distinction between unexpected psychological feature radio networks, wireless sensing element networks, and psychological feature radio wireless sensing element networks, potential application areas of psychological feature radio wireless sensing element networks, challenges and analysis trend in psychological feature radio wireless sensing element networks. The sensing schemes suited to psychological feature radio wireless sensing element networks situations ar mentioned with a stress on cooperation and spectrum access strategies that make sure the accessibility of the desired QoS.

Xun Li et al. (2013) outlined that potential for a cooperative, sturdy network of microsensors has attracted a good deal of analysis attention. For the foremost half, this can be thanks to the compelling applications which will be enabled once wireless micro-sensor networks ar in place; location-sensing, environmental sensing, medical observance and similar applications ar all gaining interest. However, wireless microsensor networks cause various style challenges. For applications requiring future, sturdy sensing, corresponding to military intelligence operation, one vital challenge is to style sensing element networks that have long system lifetimes. This challenge is particularly tough thanks to the energy strained nature of the devices. so as to style networks that have extraordinarily long lifetimes, author projected a physical layer driven approach to coming up with protocols and algorithms.

Karim Seada et al. (2013) outlined that the large-scale preparation of wireless sensing element networks (WSNs) and therefore the want for knowledge aggregation necessitate economical organization of the constellation for the aim of equalisation the load and prolonging the network lifespan. The past few years have witnessed hyperbolic interest within the potential use of wireless sensing element networks (WSNs) in applications corresponding to disaster management, combat field intelligence operation, border protection and security police work. it absolutely was studied that the bunch strategies in WSN that improve energyefficiency and discuss the look principle of the various bunch approaches, classify the projected approaches supported their objectives and style principles.

Neeraj Kumar Mishra et al. (2012) projected S-MAC, a medium-access management (MAC) protocol designed for wireless sensing element networks. Wireless sensing element networks use battery-operated computing and sensing devices. A network of those devices can collaborate for a typical application corresponding to environmental observance. it absolutely was expected the sensing element networks to be deployed in an advertisement hoc fashion, with individual nodes remaining for the most part inactive for long periods of your time, on the other hand turning into suddenly active once one thing is detected. These characteristics of sensing element networks and applications encourage a macintosh that's totally different from ancient wireless MACs corresponding to IEEE 802.11 in nearly each way: energy conservation and selfconfiguration ar primary goals, whereas per-node fairness and latency ar slighter. S-MAC uses 3 novel techniques to scale back energy consumption and support self-configuration. to scale back energy consumption in taking note of associate degree idle channel, nodes sporadically sleep. Neighboring nodes type virtual clusters to auto-synchronize on sleep schedules. galvanized by PAMAS, S-MAC conjointly sets the radio to sleep throughout transmissions of alternative nodes.

Prakashgoud Patil et al. (2013) outlined WSNs with MEs and supply a comprehensive taxonomy of their architectures, supported the role of the MEs and so gift an summary of the info assortment method in such situation, and establish the corresponding problems and challenges. Wireless sensing element networks (WSNs) have emerged as an efficient answer for a large vary of applications. Most of the standard WSN architectures carries with it static nodes that ar densely deployed over a sensing space. Recently, many WSN architectures supported mobile parts (MEs) are projected. Most of them exploit quality to deal with the matter of information assortment in WSNs.

Sanjay Eknath Gawali et al.(2013) This paper introduced a set of mobile wireless sensing element networks, referred to as smart-phone sensing element networks, wherever giant numbers of smartphone devices work to perform sensing tasks. whereas these rising networks show high potential, very little work has been done on design-time verification and validation to make sure that a designed system can meet the required goals. This paper introduces Empower, a simulation setting for smart-phone sensing element networks that simulates smart-phone-specific properties of a sensing element network, corresponding to knowledge assortment policies, and outputs high-level system metrics, corresponding to coverage of the setting being monitored.

Neeraj Kumar et al. (2012) projected a completely unique approach for the mobile users to gather knowledge network wide. Most of the standard Wireless sensing element Network (WSN) architectures carries with it static nodes that ar densely deployed over a sensing space. The route structure of information assortment is updated on every occasion once the movement of the mobile user changes. By considering this approach we have a tendency to perform restricted modification to update the route structure whereas the route performance is finite and controlled compared to the optimum performance within the Wireless sensing element Network. during this we'd like to update the route structure of information assortment whenever there's mobile movement. The projected protocol to update route structure is simple to implement. The protocol uses 3 approaches as: low-level formatting of information assortment tree, Updation of information assortment tree, Routing of information. Paper analysis generated the higher than mentioned protocol is use to shows that the projected approach is ascendible in maintenance overheads, performs with efficiency within the routing and provides continuous knowledge delivery throughout the movement of the user.

N. Akilandeswari et al. (2013) during this paper, a replacement protocol referred to as equal Cluster Head Election Routing Protocol (ECHERP), that pursues energy conservation through balanced bunch, is projected. ECHERP models the network as a linear system and, victimization the Gaussian elimination algorithmic program, calculates the combos of nodes which will be chosen as cluster heads so as to increase the network lifespan. The performance analysis of 
ECHERP is allotted through simulation tests, that impart the effectiveness of this protocol in terms of network energy potency in comparison against alternative well-known protocols.

Mohit Saini et al. (2013) As sensing element nodes ar typically powered devices, the vital aspects to face concern the way to scale back the energy consumption of nodes, in order that the network lifespan will be extended to cheap times. during this paper author initial break down the energy consumption for the parts of a typical sensing element node, and discuss the most directions to energy conservation in WSNs. Then, we have a tendency to gift a scientific and comprehensive taxonomy of the energy conservation schemes, that ar afterwards mentioned thorough. Special attention has been dedicated to promising solutions that haven't however obtained a large attention within the literature, corresponding to techniques for energy economical knowledge acquisition.

\section{PERFORMANCE METRICS}

In this research different scenarios are taken into consideration with varying number of nodes against constant simulation time. Comparison is drawn between two coverage techniques on the basis of delay, load, throughput, data dropped and retransmission attempts.

Simulation Time: The time taken for each simulation to run or it can be said as the time between start and end of it.

Delay: Delay of network specified how long it takes for a bit/packet of data to travel across the network from one node to another.

Load: It refers to the amount of data that is carried by a network. It is expressed as bits/sec or packets/sec.

Throughput: It is an average rate of successful message delivery over a network. It is measured in Bits/sec or packets/sec.

Data Dropped: It is the amount of data that is not received to the destination and is dropped from the network. It is expressed in bits or packets.

Retransmission Attempts: It is the number of attempts that is taken by a source to deliver a message to the destination. It is represented in bits/sec.

During preliminary study, it has been studied that for creating any network some assumptions are taken into account. For the implementation of coverage techniques in WSN, simulation parameters used are shown in Table below;

Table 1: Simulation Parameters

\begin{tabular}{|c|c|}
\hline Simulation Parameter & Value \\
\hline Frame duration & 1.0 millisecond \\
\hline Frequency Bandwidth & $25 \mathrm{MHZ}$ \\
\hline Mode of transmission & TDD \\
\hline $\begin{array}{c}\text { Number of mobile } \\
\text { station }\end{array}$ & $20,40,60$ \\
\hline Packet size & $5 \mathrm{~kb}$ \\
\hline Simulation grid size & $100 \mathrm{~m} \mathrm{X} \mathrm{100} \mathrm{m}$ \\
\hline Simulation time & $125 \mathrm{sec}$ \\
\hline
\end{tabular}

\section{METHODOLOGY}

PDORP: Initially, in this stage, all the sensor nodes are randomly placed in the environment depends on the application. All nodes in the network compute its hop count distance from the BS. For calculation of hop count, BS broadcasts the Beacon message containing its identity. The node, which receives the Beacon signal responds with its id and its location coordinates. BS calculate the hop count from each node using the node coordinates and send the hop count value to nodes. Each sensor node stores hop count value in Neighbor Node information table (NN table). The NN table consist of Next-Node, Hop Count between Next node and BS (HC-BS), Neighbor Node Remaining Energy (NNRE), Distance (Distance between source node and next node), and Distance from next node to BS (D-to-BS). To calculate the neighbor node information, source nodes (Src-ID) sends a REQUEST packet to the neighboring nodes. The neighboring (Dest-ID) node upon receiving REQUEST packet, search in its NN table for HC-BS, NNRE, and Coordinates. Then, it replies with the REPLY packet to the source node (Src-ID) then source node updates its NNtable. The format of REQUEST and REPLY packets.

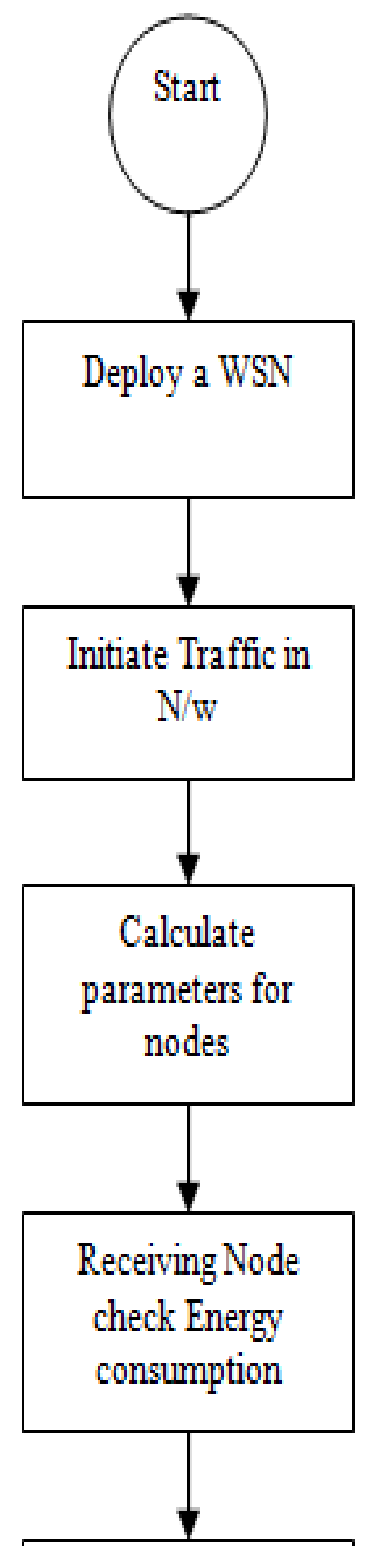




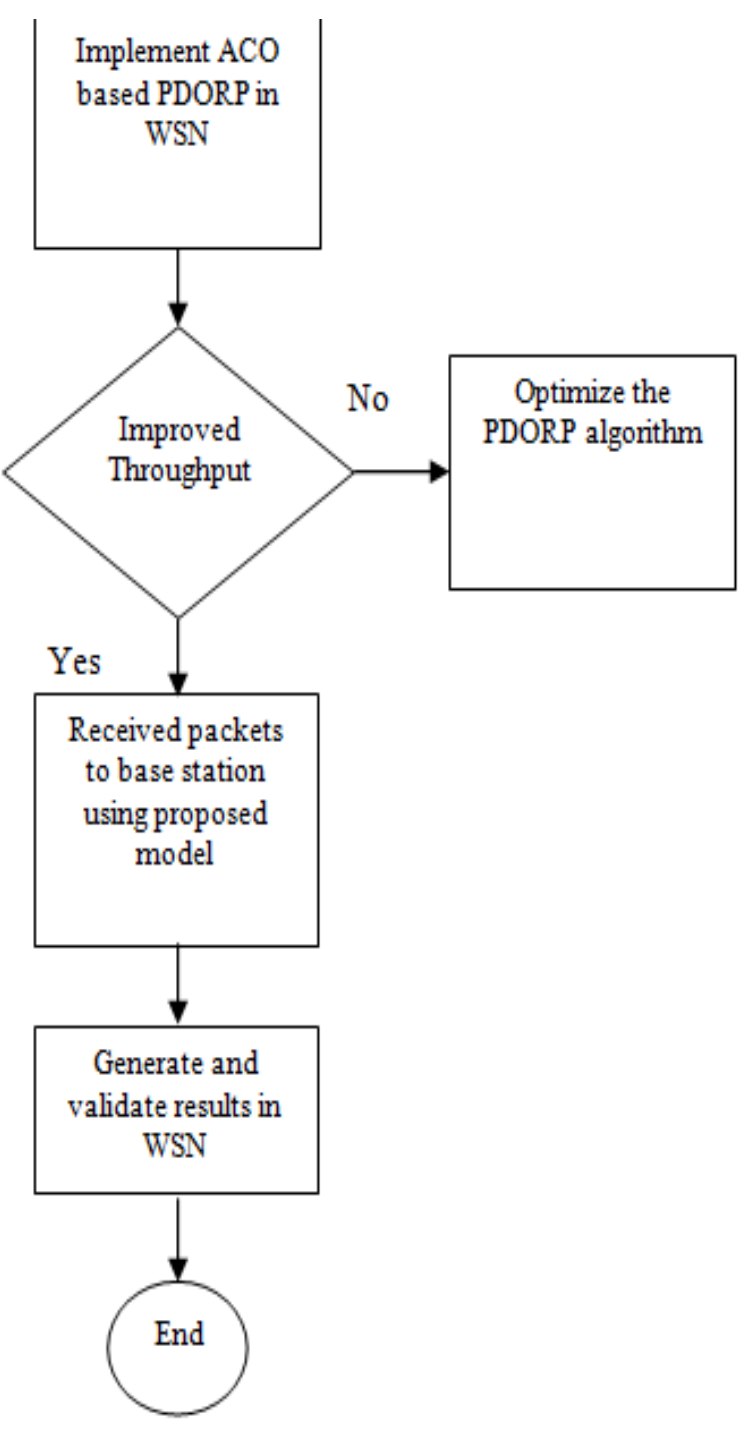

\section{RESULTS AND DISCUSSION}

In current research with the help of comparative study, we can draw all the pros and cons of the above defined scheduling schemes. In this scenario a comparison is made between hybrid routing schemes which is shown below.

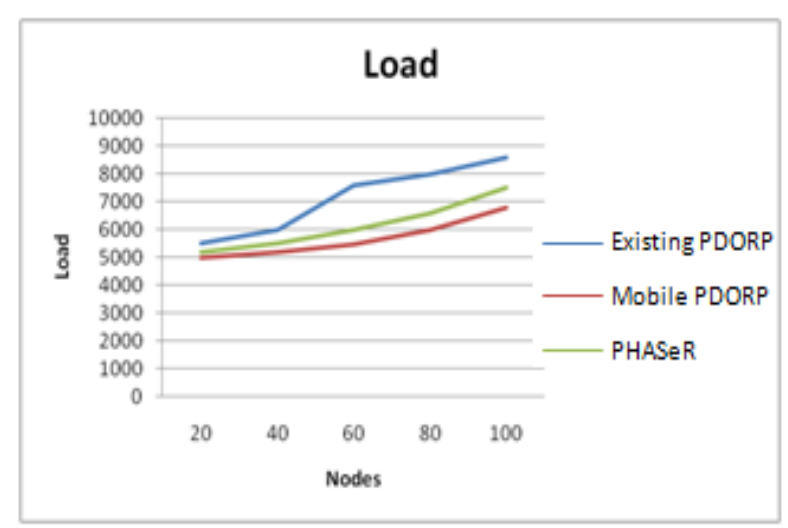

Fig 2: Average load

Load: The load in two WSN protocols called WSN base and PDORP in 20, 40, 60, 80 and 100 nodes. From the above graph it is shown that the load in proposed approach is less than that of existing approach. Average load in case of proposed approach is approx 6Kbits where as in case of existing approach it is 7 Kbits.

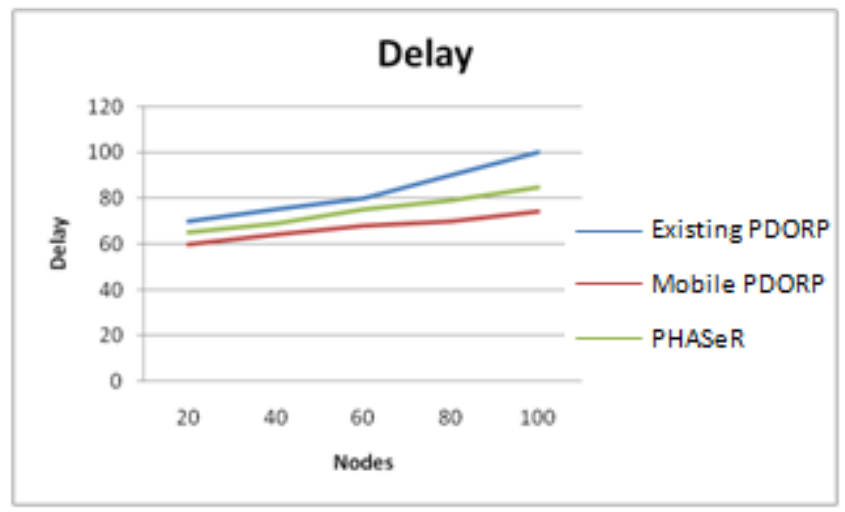

Fig 3: Delay

Delay: Delay in PDORP and WSN in 20, 40, 60, 80, 100 nodes. From the graph it can easily depicted that the delay in PDORP is less than that of existing WSN protocol. End to end delay in case of proposed approach is approx $90 \mathrm{msec}$ where as in case of existing approach it is $110 \mathrm{msec}$.

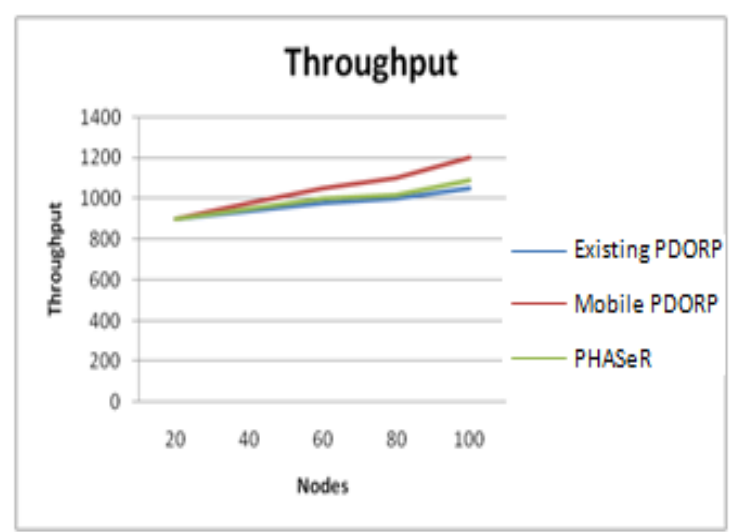

Fig 4: Throughput

Throughput: Throughput in PDORP and WSN in 20,40, 60, 80,100 nodes. From the graph it can easily depicted that the throughput in PDORP is less than that of existing WSN protocol. Throughput in case of proposed case is approx 1200 packets and in existing case it is approx 1000 packets.

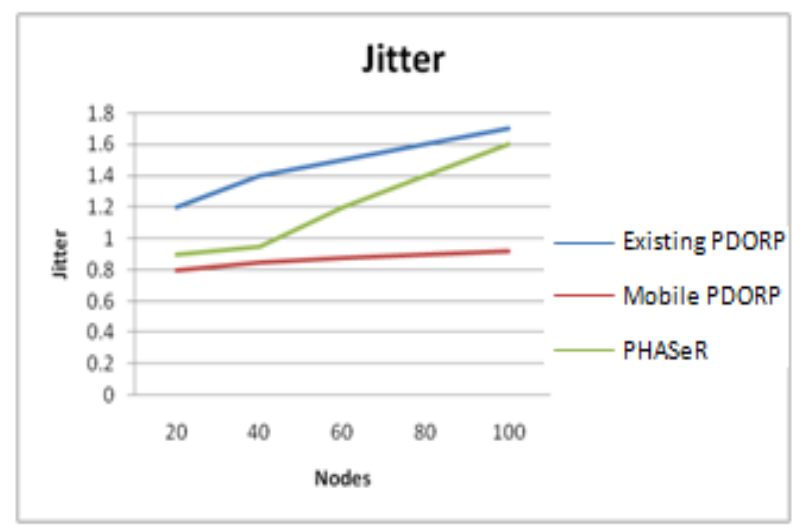

Fig 5: Jitter

Jitter: Jitter in PDORP and WSN in 20,40,60,80,100 nodes. From the graph it can easily depicted that the jitter in PDORP is less than that of existing WSN protocol. Jitter in case of proposed case is approx $1.3 \mathrm{sec}$ and in existing case it is approx $1.4 \mathrm{sec}$. 


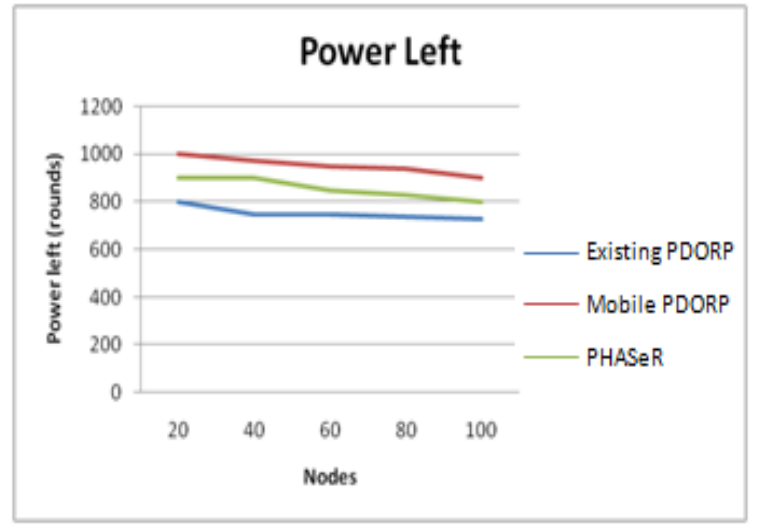

Fig 6: Power Left

Power Left: Power Residual in PDORP and WSN in 20, 40, $60,80,100$ nodes. From the graph it can easily depicted that the residual power in PDORP is more than that of existing WSN protocol. Residual power in case of proposed case is retained upto approx 900 rounds and in existing case it is approx 750 rounds.

Table 2: Comparative study for existing and proposed results

\begin{tabular}{|l|c|c|c|}
\hline \multicolumn{1}{|c|}{ Algorithm } & $\begin{array}{c}\text { Existing } \\
\text { PDORP }\end{array}$ & $\begin{array}{c}\text { Dynamic } \\
\text { PDORP }\end{array}$ & $\begin{array}{c}\text { Mobile } \\
\text { PHASeR }\end{array}$ \\
\hline Delay(sec) & 90 & 110 & 100 \\
\hline Throughput(packets) & 1000 & 1200 & 1050 \\
\hline Residual power & 750 & 900 & 800 \\
\hline Jitter(sec) & 1.4 & 1.3 & 1 \\
\hline $\begin{array}{l}\text { Average } \\
\text { Load(bits/sec) }\end{array}$ & 7000 & 6000 & 6600 \\
\hline
\end{tabular}

\section{CONCLUSION}

In the proposal an approach is defined for energy efficiency and reduction in data dropped in the WSN network. In PDORP algorithm a subset is defined, in which if a single node in that subset is died out then the whole subset have to be replaced. A modification in the PDORP algorithm is made to achieve the objectives of the research in which an energy threshold set for each node. In case of the proposed approach with the low energy in the subset of network the neighbor nodes of the subset can cover the area of the died node. In this way the data dropped due to the replacement of the subset may be reduced so there is a reduction in the energy dissipation. Using this approach the data dropped and other Quality parameters are also improved like, delay, load and throughput etc as defined in results and discussion.

\section{FUTURE SCOPE}

In the future scope of this study the replacement cost of the subset in network can be reduced. The scalability of the approach can be improved in a way so that quality parameters can not be reduced. Improvement can be shown in case where the maximum number of subsets is to generate in PDORP algorithm but the data dropped can should be reduced. This study may be improved in terms of scalability also. One can check the algorithm on various set on nodes without compromising the Quality of Service parameters. On the other hand the security issues may be incorporated in this research as a future scope. To transfer the data from source to destination an encryption algorithms may be applied for secure communication.

\section{REFERENCES}

[1] Guravaia; K.; Velusamy, R. L.; "PDORPMRP: River Formation Dynamics based Multi-Hop Routing Protocol for Data Collection in Wireless Sensor Networks", Eleventh International Multi-Conference on Information Processing, Volume: 54, 2015, pp: 31-36

[2] Joshi, G. P.; Nam, S. Y.; Kim, S. W.; "Cognitive Radio Wireless Sensor Networks: Applications, Challenges and Research Trends", Sensors, ISSM: 1424-8220, Volume: 13, No: 9, Aug 12, 2013, pp: 11196-11228

[3] Li, X; Merrett, G. V.; White, N. M.; "Energy-efficient data acquisition for accurate signal estimation in wireless sensor networks", Journal on wireless Communications and Networking, Volums: 230, Issue:1, 2013, pp: 1-15

[4] Seada, K.; Zuniga, M.; Helmy, A.; Krishnamachari, B.; "Energy Efficient Forwarding Strategies for Geographic Routing in Lossy Wireless Sensor Networks", Proceedings of the 2nd international conference on Embedded networked sensor systems, USA, ISBN:158113-879-2, 2004, pp: 108-121

[5] Mishra, N. K.; Jain; V.; Sahu, S.; "Survey on Recent Clustering Algorithms in Wireless Sensor Networks", International Journal of Scientific and Research Publications, ISSN 2250-3153, Volume: 3, Issue: 4, April 2013, pp: 1-4

[6] Patil, P.; Kulkarni, U. P.; "Energy Efficient Aggregation With Divergent Sink Placement For Wireless Sensor Networks", International Journal of Ad hoc, Sensor \& Ubiquitous Computing (IJASUC), Volume: 4, No.2, April 2013, pp: 47-58

[7] Gawali, S. E.; Mantri, D. S.; "Lifetime Energy Efficient Optimization for WSN", 2nd International Conference, Computer Technology and Development, E-ISBN :9781-4244-8845-2, Print ISBN:978-1-4244-8844-5, 2010, pp: $235-239$

[8] Kumar, N.; Kumar, M.; Patel, R. B.; "A Secure and Energy Efficient Data Dissemination Protocol for Wireless Sensor Networks", International Journal of Network Security, Volume.15, No.6, 2012, pp.490-500.

[9] Akilandeswari, N.; Santhi B.; Baranidharan, B.; "A Survey on Energy Conservation Techniques In Wireless Sensor Networks", ARPN Journal of Engineering and Applied Sciences, ISSN 1819-6608, Volume. 8, No. 4, APRIL 2013, pp: 265-269

[10] Saini, M.; Saini, R. K.; "Solution of Energy-Efficiency of sensor nodes in Wireless sensor Networks", International Journal of Advanced Research in Computer Science and Software Engineering, ISSN: 2277 128X, Volume 3, Issue 5, May 2013, pp: 353-357

Khan, A. W.; Abdullah, A. H.; Razzaque, M. A.; Bangash, J. I.; "VGDRA: A Virtual Grid-Based Dynamic Routes Adjustment Scheme for Mobile Sink-Based Wireless Sensor Networks", IEEE Sensors Journal, Volume: 15, Issue: 1, January 2015, pp: 526-534

[11] Long, J.; Dong, M.; Ota, K.; Liu, A.; Hai, S.; "Reliability Guaranteed Efficient Data Gathering in Wireless Sensor 
Networks", IEEE, ISSN: 2169-3536, Volume: 3, 2015, pp: 430-444

[12] Prabha, K. A.; Hemapriya, K.; "Energy Saving In Wireless Sensor Network Using Optimal Selective Forwarding Protocol", International Journal of Advancements in Research \& Technology, Volume: 2, Issue: 1, January-2013, pp: 1-6

[13] Hayes, T.; Ali, F.H.; "Proactive Highly Ambulatory Sensor Routing (PHASeR) protocol for mobile wireless sensor networks", Pervasive and Mobile Computing, Volume: 21, 2015, pp: 47-61

[14] Singh, S.; Meenaxi, "A Survey on Energy Efficient Routing in Wireless Sensor Networks", International Journal of Advanced Research in Computer Science and Software Engineering, ISSN: 2277-128X, Volume 3, Issue 7, July 2013, pp: 184-189. 